\title{
The golden ratio in facial symmetry*
}

\author{
Emmanuel P. Prokopakis' ${ }^{1}$ loannis M. Vlastos' ${ }^{1}$, Valerie Picavet ${ }^{2}$, Gilbert Nolst \\ Trenité ${ }^{3}$, Regan Thomas ${ }^{4}$, Cemal Cingi ${ }^{5}$, Peter W. Hellings ${ }^{6}$ \\ ' Department of Otorhinolaryngology, University of Crete School of Medicine, Heraklio, Crete, Greece \\ ${ }^{2}$ Department of Otorhinolaryngology, University Hospital UIm, UIm, Germany \\ ${ }^{3}$ Department of Otorhinolaryngology, AMC University of Amsterdam, Amsterdam, the Netherlands \\ ${ }^{4}$ Department of Otolaryngology - Head and Neck Surgery, University of Illinois, Chicago, IL, USA \\ ${ }^{5}$ Department of Otorhinolaryngology, Osmangazi University, Eskisehir, Turkey \\ ${ }^{6}$ Department of Otorhinolaryngology, University Hospitals Leuven, Leuven, Belgium
}

\section{SUMMARY}

Symmetry is believed to be a hallmark of appealing faces. However, this does not imply that the most aesthetically pleasing proportions are necessary those that arise from the simple division of the face into thirds or fifths. Based on the etymology of the word symmetry, as well as on specific examples and theories of beauty, we conclude that $\varphi$-value, a ratio also known as the golden ratio or the divine proportion, can also characterize symmetrical forms. Therefore, we propose the utilization of this ratio in facial aesthetics.

Key words: face, symmetry, beauty, golden ratio

\section{Introduction}

Mankind has always attempted to define beauty and to elucidate key elements of facial aesthetics ${ }^{(1)}$. From ancient Greek times through the Renaissance to modern surgical practice, the classical position that attributes beauty to specific symmetrical proportions has been in doubt. This doubt results from the erroneous definition of symmetry as a precise and well-defined concept of balance, or 'patterned self-similarity.'

The Greek philosopher Aristotle defined beauty as 'an imprecise sense of harmonious or aesthetically pleasing proportionality ${ }^{(2)}$. Since then, a variety of aesthetic proportions have been proposed, such as the 1:1 ratio, and the axial facial division into thirds ${ }^{(3)}$. Though, it seems that the quantitative characteristics of beauty are best being elucidated when the 'golden ratio' is employed as the most aesthetically pleasing proportion. The 'golden ratio' (Figure 1a) is a proportion very commonly seen in nature, and it has long been proposed as a beautiful character- istic of natural and artistic creations. The golden ratio divides a line at a point, such that the ratio of the lengths of the two sides $(a / b)$ is equal to the ratio of the sum of the two sides $(a+b)$ to the longer side (a) (Figure 1). Herein, we propose the utilization of the golden ratio as the most appealing symmetrical form, or in other words the 'metron' that characterizes attractive faces.

\section{Metron, symmetry and metron}

Symmetry, and as a consequence beauty, is not necessarily defined as 'patterned self similarity' or an entity of mirror imaged parts. Etymologically it is related to the application of a 'metron.' Metron is regarded as the smallest unit or as a measure of both quantitative and qualitative parameters. It is utilized in determining the magnitude of quantity, or the limits of a non-measurable feature ${ }^{(4)}$.

According to the etymology of the word symmetry ('syn' + 'metron' = in correspondence to metron), an object is considered 
to be symmetrical when it is characterized by specific measures or proportions, irrespectively whether it retains a constituent configuration on opposite sides, around a center or an axis. In other words, symmetry is defined as 'a relationship of characteristic correspondence or equivalence among constituents of an entity or between different entities ${ }^{(4)}$, if the 1:1 ratio (or the so called unity ratio) is used as a 'metron.' However, broader definitions of symmetry have been suggested such as 'correct or pleasing proportion of the parts of a thing' (5) or 'beauty as a result of balance or harmonious arrangement ${ }^{\prime(5)}$. These are more acceptable explanations in terms of the word etymology. Symmetry in human faces has been suggested to be a cue to heritable fitness benefits, and related to sexual dimorphism ${ }^{(6)}$. In general, the degree of symmetry in signals is believed to indicate the signaler's quality. By contrast, several experiments using artificial neural networks have shown that symmetry preferences may arise as a by-product of the need to recognize objects irrespective of their position and orientation in the visual field ${ }^{(6)}$. Moreover, female preference for symmetrical males is a by-product of selection for mate recognition ${ }^{(7)}$. Independently from the preferred theory, biological evolution and artistic innovation directs a convergence in symmetrical forms. The 1:1 ratio or the so called 'unity ratio' is the most easily identifiable proportion. However, when it comes to aesthetical pleasing analogies, it is the 'golden ratio' that has long been credited as the most alluring proportion. In other words, apart from the unity ratio, the most common symmetrical form being observed in our natural and cultural environment, from flowers and leaflets' veins to classical buildings, sculptures and paintings is the 'golden ratio.'

\section{Golden ratio as metron in nature and art}

The precise value of the golden ratio is the irrational number phi $(\Phi=a / b=(a+b) / a=1.61803399 . .$.$) named by the sculptor$ of the Parthenon Phidias. The golden ratio is also referred to as the Fibonacci ratio or the 'divine proportion'. Several examples of the presence of the phi ratio can be found in both nature and art (Figure 1). Its' mathematical properties have been described in detail by the ancient Greeks ${ }^{(8)}$ although it seems that they were already known for centuries by other civilizations, such as the Egyptians. Over the years, several Western intellectuals of diverse background, from architecture to medicine and from arts to philosophy, have been fascinated by the 'divine proportion'. However, a lot of debate has raged as to its aesthetic qualities. The use of this proportion, especially in art and architecture, and generally in the industry of beauty, has been both advocated and condemned ${ }^{\left({ }^{6}\right)}$. Moreover, it is supported that the aesthetic notion of beauty is vague, subjective and may not be reducible to a few simple ratios. Nevertheless, ' $\varphi$ ' can characterize a symmetrical form, considering the aforementioned definition of symmetry, as beauty of form arising from balanced proportions not necessary reduced to mirror images.

\section{Quantifying facial aesthetics}

Philosophers and scientists have long tried to appreciate beauty in terms of numeric symmetry and proportions, mostly by dividing the face into quadrants or thirds ${ }^{(3)}$ (Figure 2, A-B). The validity of these schemes has not been verified by modern analysis ${ }^{(3,10)}$, enhancing the notion that beauty is an individual, cultural and non-quantifiable matter. However, one can not dispute that the aesthetic perfection of the face is not an abstract conception, but rather a quantitatively well-defined anatomic quality ${ }^{(11)}$. Aesthetic judgments of the shape of a person's face are similar across different cultural backgrounds ${ }^{(11)}$ and different ages ${ }^{(12)}$. As long as an inter-observer concordance regarding facial beauty can be reached, norms of measurements and proportion indices will continue to be studied in attractive faces, and used as a measure of objective beauty. Since several parts of living organisms, man made structures and dynamic entities seem to conform to the golden ratio, it was rational for surgeons to propose this ratio as a planning tool for the reconstruction of facial deformities ${ }^{(13,14)}$.

Numerous facial landmarks and proportions, like eye width/ mouth width, menton- nasion/menton-trichion, as well as other corporal ratios have been proposed ${ }^{(3)}$. However, the majority of them, like nose width/mouth width, nose width/nose length eye width/mouth width and dental width/dental height, some times are not considered ideal unless they conform to the golden ratio ${ }^{(15,16)}$. Nevertheless, several methodological issues have not been addressed adequately in these studies. Average proportions have been used, although highly attractive facial configurations are not average ${ }^{(17)}$, and the complexity of facial configurations, like repose or smiling positions and other expressions have been ignored ${ }^{(18)}$. To date, only very few facial landmarks and proportions have been shown to influence consistently the perception of attractiveness. Trichion, menton, nasion and subnasale are among those that are consistently being proposed or employed by researches of facial beauty ${ }^{(19)}$. Interestingly, proportions that arise by them seem to conform to the golden ratio in more attractive faces ${ }^{(20,21)}$. Examples of these proportions are depicted in Figure $2 \mathrm{~A}$, and can be considered as simplified modifications of a facial mask that was proposed several years ago. This mask has been used as a reference template for facial characteristics and it was based on phi values ${ }^{(22,23)}$. Dynamic parameters, such as the smiling configuration or the processes of aging, can also be addressed. For example, the eye width to mouth width ratio is shifting away from the golden ratio in the repose position in contrast to the smiling position or in the aging face by either a relative decrease of mouth width, or increase of orbital aperture width respectively ${ }^{(23,24)}$. 


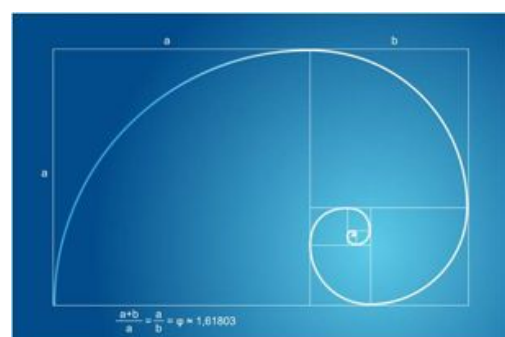

A

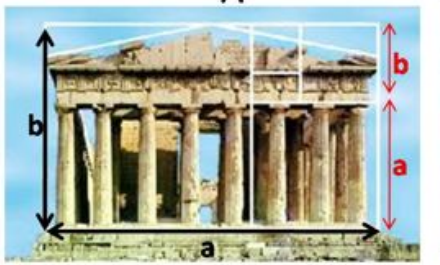

C

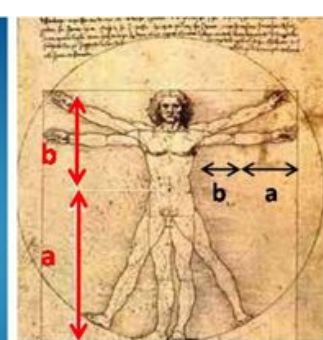

B

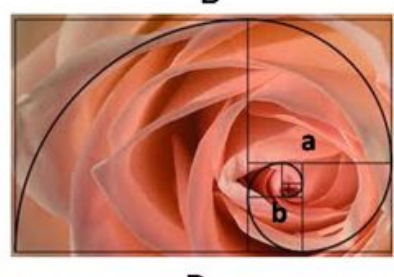

D
Figure 1. Examples of phi ration in nature and art. In all cases a:b should equal $1.61 \ldots$

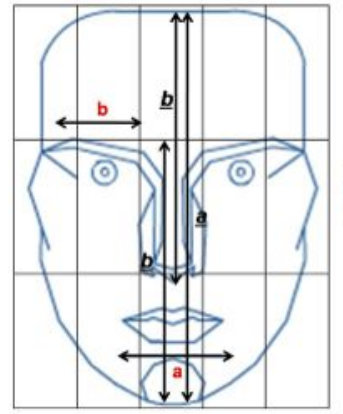

2A

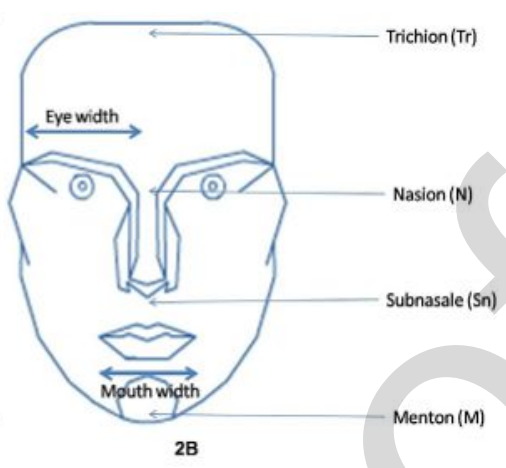

28
Figure 2. (A) Facial graph, divided vertically into fifths and horizontally into thirds as proposed by neoclassical canons9. (B) Proportions that conform to golden ratio $(\varphi=a: b)$ in beautiful faces are (Tr-M):(Tr-Sn), (Tr-M):(N-M) in repose position, and eye width: mouth width in smiling position.

\section{Phi value novelty and implications}

Facial beauty enhances self-esteem and social integration. Beautiful faces are better remembered and this has been reflected by greater interaction between a region associated with reward, the orbito-frontal cortex, and a region associated with successful memory encoding, the hippocampus ${ }^{(25)}$. Reliable reference templates that can serve as universal standards of facial beauty have not yet been accomplished.

The human face is a bewildering source of information (26). Cultural influences, masculine-feminine characteristics, aging process, subtleties of light that change with the orientation of the face ${ }^{(27)}$, repose, smiling and other configurations endow a difficult quantifiable uniqueness to each particular face. Facial analysis has moved onto sophisticated grounds, and it seems that the Phi ratio demonstrates several difficulties in explaining complex issues such as facial harmony in the moving face, inter-racial mixtures and its effect on the concept of beauty, as well as the racial connotation of altering features.

The establishment of a universal standard for facial beauty will significantly simplify the diagnosis and treatment of facial disharmonies and abnormalities. Current technology allows for a better conception of facial beauty, by utilization of a combination of techniques, such as morphing software ${ }^{(19)}$, systematic mapping, three-dimensional evaluation of the four levels of facial structure (bone, muscle, fat, and skin) ${ }^{(28)}$ and others. Specific ratios, such as the golden ratio, deserve further attention since surgical and technological evolution constantly increases the possibility of accomplishing these ratios down to one millimeter. The suggestion of using the phi value, from architecture or art to facial plastic surgery, is not a new one. The novelty relates to the concept that the phi ratio as a measure or metron should be regarded as a symmetrical form. In more details, as it can be conceptualized by the definition of symmetry, a symmetrical hence an appealing object or face should not necessarily conform to a self-similarity pattern, or to the unity ratio. When specific ratios conform to the golden ratio, then by definition the face can be considered symmetrical. The importance of the conception that this kind of symmetrical form defines facial beauty remains to be clarified.

The notion that 'beauty is on the eyes of the beholder' can be generally regarded as a neutralized and prudent remark. Though, facial plastic surgeons, otorhinolaryngologists, dermatologists, maxillofacial surgeons, orthodontists and other physicians working on facial aesthetics, in case they are being asked for normative values and aesthetics comments, could suggest that facial proportions defined by the $\varphi$ number can be considered symmetrical and appealing.

\section{Author contribution}

EPP has the idea, EPP and IMV wrote the initial draft, VP, GNT, RT, CC and PWH revised the initial draft.

\section{Conflict of interest}

No financial disclosures or other conflict of interest is reported. 


\section{REFERENCES}

1. Thomas JR. More than a pretty face: The Mona Lisa. Arch Facial Plast Surg 2008;10(1):65-66

2. Aristotle. Nicomachean Ethics (1094a)

3. Farkas LG, Hreczko TA, Kolar JC, Munro IR. Vertical and horizontal proportions of the face in young adult North American Caucasians: revision of neoclassical canons. Plast Reconstr Surg. 1985; 75: 328-338.

4. The American Heritage Dictionary of the English Language, 4th Edition, Houghton Mifflin Company. 2000

5. Simpson J. Oxford English Dictionary. 2nd edition

6. Little AC, Jones BC, Waitt C, Tiddeman BP, Feinberg DR, et al. Symmetry Is Related to Sexual Dimorphism in Faces: Data Across Culture and Species. PLoS ONE. 2008; 3 e2106.

7. Johnstone RA. Female preference for symmetrical males as a by-product of selection for mate recognition. Nature 1994; 372: 172 175.

8. Euclid, Elements, Book 6, Definition 3.

9. Enquist M., Arak A. Symmetry, beauty and evolution. Nature. 1994; 372: 169-172.

10. Bruce W. Baker, Michael G.Woods. The role of the divine proportion in the esthetic improvement of patients undergoing combined orthodontic/orthognathic surgical treatment. Int J Adult Orthod Orthognath Surg. 2001; 16: 108-120.

11. Farkas LG, Kolar JC. Anthropometrics and art in the aesthetics of women's faces. Clin Plast Surg. 1987; 14: 599-616.

12. Samuels CA, Butterworth G, Roberts T,
Graupner L, Hole G. Facial aesthetics babies prefer attractiveness to symmetry. Perception. 1994; 23: 823-831.

13. Seghers MJ, Longacre JJ, de Stefano GA The golden proportion and beauty. Plast Reconstr Surg. 1964; 34: 382-386.

14. Ricketts RM. The biologic significance of the divine proportion and Fibonacci series. Am J Orthod 1982; 81: 351-370.

15. Moss JP, Linney AD, \& Lowey MN. The use of three-dimensional techniques in facial esthetics. Semin Orthod 1995; 1: 94-104.

16. Rosenstiel SF, Ward DH, Rashid RG. Dentists' preferences of anterior tooth proportion: A Web-based study. J Prosthodont 2000; 9: 123-136.

17. Perrett DI, May KA, Yoshikawa S. Facial shape and judgements of female attractiveness. Nature. 1994; 368: 239-242.

18. Preston JD. The golden proportion revisited J Esthet Dent. 1993; 5: 247-251.

19. Karimi K, Devcic Z, Avila D, Popenko N, Wong B. A new approach in determining lateral facial attractiveness. Laryngoscope 2010; 120 Suppl 4: S157.

20. Jahanbin A, Basafa M, Alizadeh Y. Evaluation of the Divine Proportion in the facial profile of young females. Indian J Dent Res. 2008; 19: 292-296.

21. Jefferson Y. Facial Beauty-Establishing a Universal Standard. IJO. 2004; 15: 1.

22. Marquardt SR, Stephen R. Marquardt on the Golden Decagon and human facial beauty. JCO 2002; 36: 339-347.

23. www.intmath.com/numbers/math-of-beau ty.php (Accessed April 7th 2011)

24. Shaw RB Jr, Katzel EB, Koltz PF et al. Aging of the facial skeleton: aesthetic implications and rejuvenation strategies. Plast Reconstr Surg. 2011; 127: 374-383

25. Tsukiura T, Cabeza R. Remembering beauty: roles of orbitofrontal and hippocampal regions in successful memory encoding of attractive faces. Neuroimage. 2011; 54: 653360.

26. Enquist M., Ghirlanda S. The secrets of faces. Nature. 1998; 394: 827-828.

27. Young PA., Sinha U., RiceDH., Stucker F. Circles of Prominence. A New Theory on Facial Aesthetics. Arch Facial Plast Surg. 2006; 8: 263-267.

28. Fitzgerald R, Graivier MH, Kane M, et al. Facia aesthetic analysis. Aesthet Surg J. 2010; 30 Suppl: 25S-27S.

Emmanuel P. Prokopakis, MD

Department of Otorhinolaryngology

University of Crete School of

Medicine

A Building, 3rd Floor

University avenue

Heraklio, Crete

71110 Greece

Tel: $+30-693-2237622$

Fax: +30-283-105 6635

E-mail: eprokopakis@gmail.com 